\title{
Exam Hall Plan Monitoring System Using QR Code
}

\section{Subbu VV*}

St. Joseph's College of Engineering \& Technology, India

Abstract
Nowadays, as the supply of smart phones becomes dynamic and a variety of functions of smart phones and
services based on internet is utilized, a wide range of applications are being developed and deployed. Smart phones
are becoming more favourite companions to users than desktops or notebooks. Despite the speedy growth of smart
phones and the application market, the exam hall plan which is currently being used is still in its primal forms. Previously
developed exam hall plan check system uses a notice board which is placed in the college. This project proposes a
system that is based on a QR code, which displays the exam hall details of the students before the beginning of each
exam. The students will need to scan the code in order to confirm their exam hall. This helps to save the time and more
ease to assemble in the scheduled manner.

Keywords: Hall plan; Exam hall monitoring; Exam hall

\section{Introduction}

Nowadays, in day to day life all are digitalized. In spite of this some of them were not till digitalized. One of the best examples is exam hall plans; currently it is sticking on the boards. Therefore, my motto is to provide an exam hall plan in a timely manner. So I planned to digitalize this in terms of using QR code and an android studio. Using android studio coding is developed for $\mathrm{QR}$ scanner. By using QR generator create the students information like register number, this is more sufficient for this project. Then the information contained QR code is going to be pasted on your ID card instead of Barcode. QR code which is pasted on your ID card is scanned by an android application which is developed by an android studio. After scanning it will display the exam hall plan of an individual and the information in the QR code is time and date of the particular exam along with row and column number which defines the seating arrangement of an individual. Even in the neck-off time we will know the exam hall plan in anytime, anywhere. This reduces the timings of an individual and avoids threats and this is the main advantage of my paper [1-4].

\section{Hardware Module}

Android mobile phone is the hardware module in my paper. So we need not to have an additional scanning device. Mobile camera is acting like a scanner which helps to decode the data which is present in the QR code. We can able to decode the QR code in any angle and afterwards the scanner is able to change an angle of future purposes.

\section{Software Module}

The software used in my paper is:

\section{Android studio.}

\section{QR generator.}

\section{Android studio}

Android studio is a software development, is the process by which new applications are created for the Android Operating Systems.

Applications are usually developed in JAVA programming language using the Android Software Development Kit (SDK), but other development environments also available. The SDK includes a comprehensive set of development tools. These include a debugger, libraries, a handset emulator based on QEMU, documentation, sample code, and tutorials.
By using this software, we can develop an application to scan the QR code which is pasted on your id card. After scanned, the application shows the respective results.

$\begin{array}{lll}\text { Level L (Low) } & : & 7 \% \text { of code words can be restored. } \\ \text { Level M (Medium) } & : & 15 \% \text { of code words can be restored. } \\ \text { Level Q (Quartile) } & : & 25 \% \text { of code words can be restored. } \\ \text { Level H (High) } & : & 30 \% \text { of code words can be restored. }\end{array}$

\section{QR generator}

QR generator is used to generate the QR codes which are going to be pasted on your id card. It is open source software and much user friendly. The Figure 1 shows after QR code after generating by QR code generator.

It is made up of black squares and white squares. Each square is called as modules. Data present in QR code is represented in terms of Bytes. Three corners are the heart of QR code. Unlike the older, onedimensional barcodes that were designed to be mechanically scanned by a narrow beam of light, a QR code is detected by a 2-dimensional digital image sensor and then digitally analyzed by a programmed processor. The processor locates the three distinctive squares at the corners of the QR code image, using a smaller square (or multiple squares) near the fourth corner to normalize the image for size, orientation, and angle of viewing. The small dots throughout the QR code are then converted to binary numbers and validated with an error-correcting algorithm. Above lists explains the approximate error correction capability at each of the four levels.

\section{Work Flow}

The QR code instead of barcode is pasted on your ID card. The QR

*Corresponding author: Subbu VV, AP/ECE, St. Joseph's College of Engineering \& Technology, Bharananganam Pravithanam Road, Kottayam, Palai, Choondacherry, Kerala, 686579, India, Tel: 04822-239722; E-mail: venkat251992@gmail.com

Received November 01, 2018; Accepted December 20, 2018; Published December 31, 2018

Citation: Subbu VV (2018) Exam Hall Plan Monitoring System Using QR Code. J Electr Electron Syst 7: 289. doi: 10.4172/2332-0796.1000289

Copyright: @ 2018 Subbu VV. This is an open-access article distributed under the terms of the Creative Commons Attribution License, which permits unrestricted use, distribution, and reproduction in any medium, provided the original author and source are credited. 


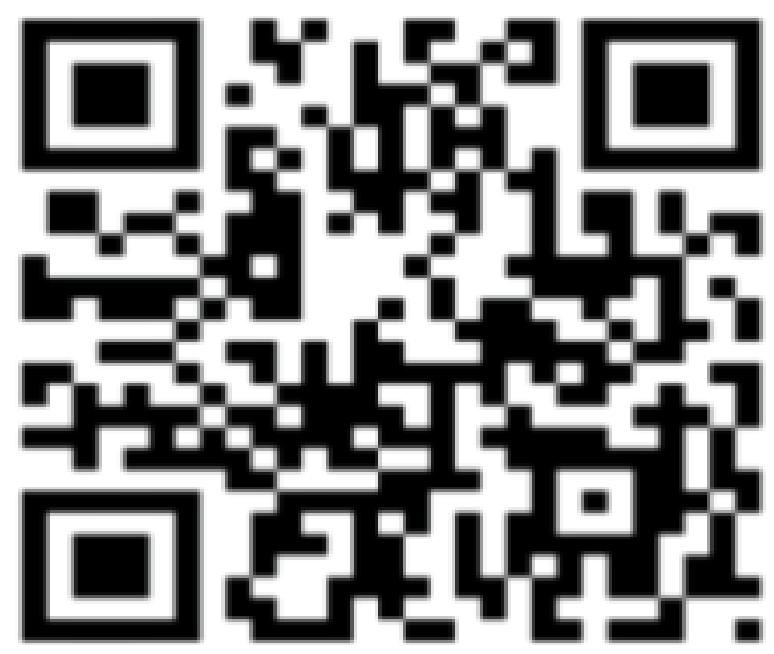

Figure 1: QR Code.

code contains the register number of an individual. Then the QR code is scanned by an image scanner that is nothing but the camera which is present on your mobile phone. The recognition process involves:

- Image binarization.

- Tilt correction.

- Geometric correction.

- Image normalization.

After recognition process, the QR code data is compared with the database. The database contains date, time, block number, seating number of an individual. If the comparison were matched it displays the results of an individual otherwise an error message will be displayed by an application (Figures 2 and 3) [5-8].

\section{Image binarization}

For more practical sense, image acquisition uses a general chat camera and apply camera control program for image acquisition under the intake image pattern. Images collected are converted to grayscale format by gray-scale processing. A good binarization method takes a very important role in the entire bar code identification system. Niblack method is the best method comparing a variety of local threshold algorithm. But it is very difficult to set an appropriate window size on the way, has great influence on the modules and also takes too much time.

\section{Tilt correction}

When the image is being scanned, the position of QR code often occurs tilt and it needs rotating operation for correction. Researchers have been done on this problem. Algorithm that hollows out internal points of the bar code then gets the edge information and peaks and slope detection of bar code with Hough transform over a known point. Look for position detection patterns before decoding by using opening operation and closing operation in morphological image processing.

However, the recognition rate of these two methods is not high. A quick location method for the QR code symbol using one-dimensional pattern match based on the specific mode of the position detection patterns of the code, and then by Hough transform applied on the edge points of the code, 4 edge lines and 4 vertices are calculated, at last the control point transform and spatial bilinear interpolation arithmetic are conducted on the code image according to the 4 vertices consisting a square.

Using Hough transform, sobel operator to locate the bar code symbol, and then uses space transformation to correct image distortion. However, the boundaries of QR code are not continuous, large errors are made by using of Hough transform positioning. Based on the constraints set by two-dimensional bar code image feature, and draws the concept of convex hull in computational geometry into locating bar code through in case of Data Matrix. This algorithm can locate twodimensional bar code effectively under the distorted or tilted situation.

\section{Image-geometric correction}

The image geometric distortion will emerge because of the Shooting angle, image rotation and other issues. QR code geometric distortion will bring great recognition errors and reduce the recognition rate. In general, the QR code image distortion is linear distortion. As a result, QR codes can be used to correct for the characteristics of being a square. Distortion correction algorithm is as follows.

1) Obtain images of the four vertices of $Q R$ codes. Due to the rotation process the external noise information has been ruled out, so we can scan QR code image line by line, from the eight directions of the QR code region (up, down, left, right, upper left, lower left, upper right, lower right) in a straight line to scan the QR code until two or more intersection between the line and black lock. After the scan at eight directions, we will

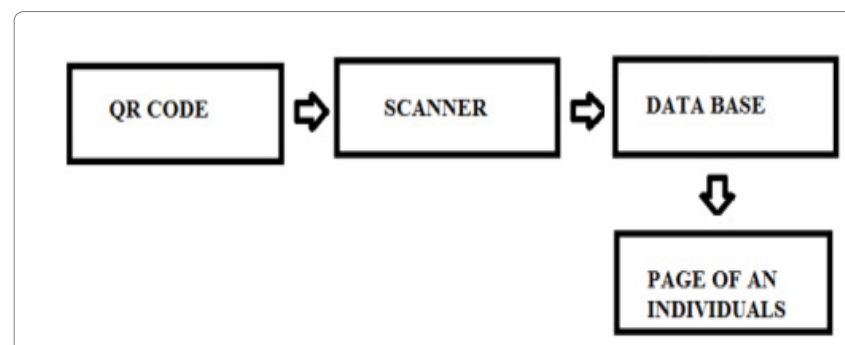

Figure 2: Block Diagram.

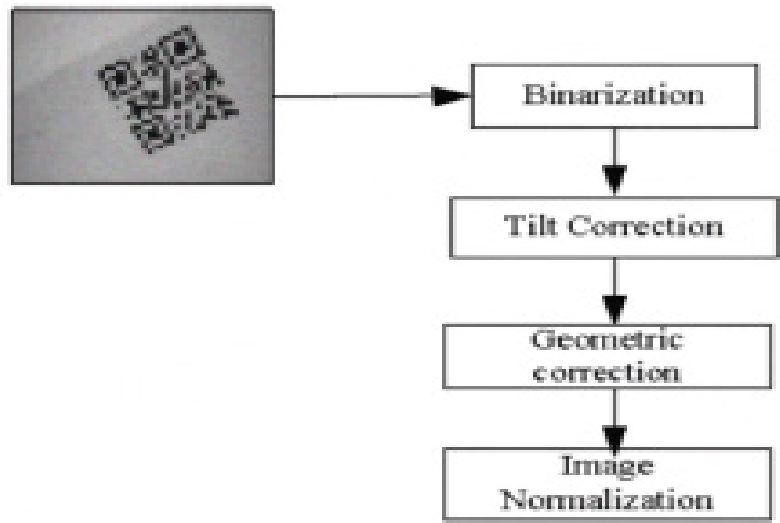

Figure 3: Processing of An Image. 
get 16 points at least; the point appeared in both directions is the vertex. When these steps are completed, there are three or more vertices. Based on the distance between vertices and the center of position detection patterns, 3 shortest vertices can be obtained and there are the vertexes.

2) Determine the fourth vertex. Algorithm that hollows out internal points of the bar code, get the edge information, with over a known point Hough transform to detect the fourth vertex of QR code. According to the known Relationship among the location of three vertexes, we can determine the Orientation of the fourth vertex in the QR code (upper left, lower left, upper right, lower right). Then the location of the adjacent two lines to the resulting the fourth vertex can be known. Scanning along the center coordinates of position detection patterns until the intersection between black line and the QR code module. The slope of the two boundary lines can be calculated. Then the point at which these two straight lines intersect is the fourth vertex.

\section{Image normalization}

The QR code image which is almost on regular can be obtained by geometric transformation. Then it needs to be normalized. The research in proposed a method that the $\mathrm{QR}$ code is divided into models based on Sobel edge detection and de blurred based on Fourier transform, and all bit stream.

Secondly, divide equally the $\mathrm{QR}$ image into $\mathrm{n} \times \mathrm{n}$ small grids according the version number, re-sample the center of each grid as the sampling point and get the normalized QR code symbol. In this process, since the computer step length is integer, the cumulative error must produce more or less. By using of averaging method, some modules that are supposed to be within the grid shift, leading to errors in $\mathrm{QR}$ codes dividing.

Therefore, it is better to make a full-scale expansion to the image before grid-dividing based on the principle of image scaling so that it minimize the error which is generated when cutting image. While the new gray values generated in amplifying procedure can be solved using bilinear interpolation. Thirdly, decode the standard QR code symbol according the National Standard Method of Quick Response Code after image re-sampling (Figure 4).

\section{Results}

Results show stages of output. The results are shown below:

\section{College Id-card}

Shows the college Id card. It contains QR code instead of Barcode. QR contains the register number of an individual (Figure 5).

\section{While scanning}

The android application scans the QR code which is pasted on your id card (Figure 6).

\section{After scanned}

Correct result: After scanned by an android application, the result will be displayed like this and it contains date, time, block number, seating number (Figure 7).

Incorrect result: If any fault or damages in an id card or other institution id card means the application shows an error message like this after scanned by the user (Figure 8).
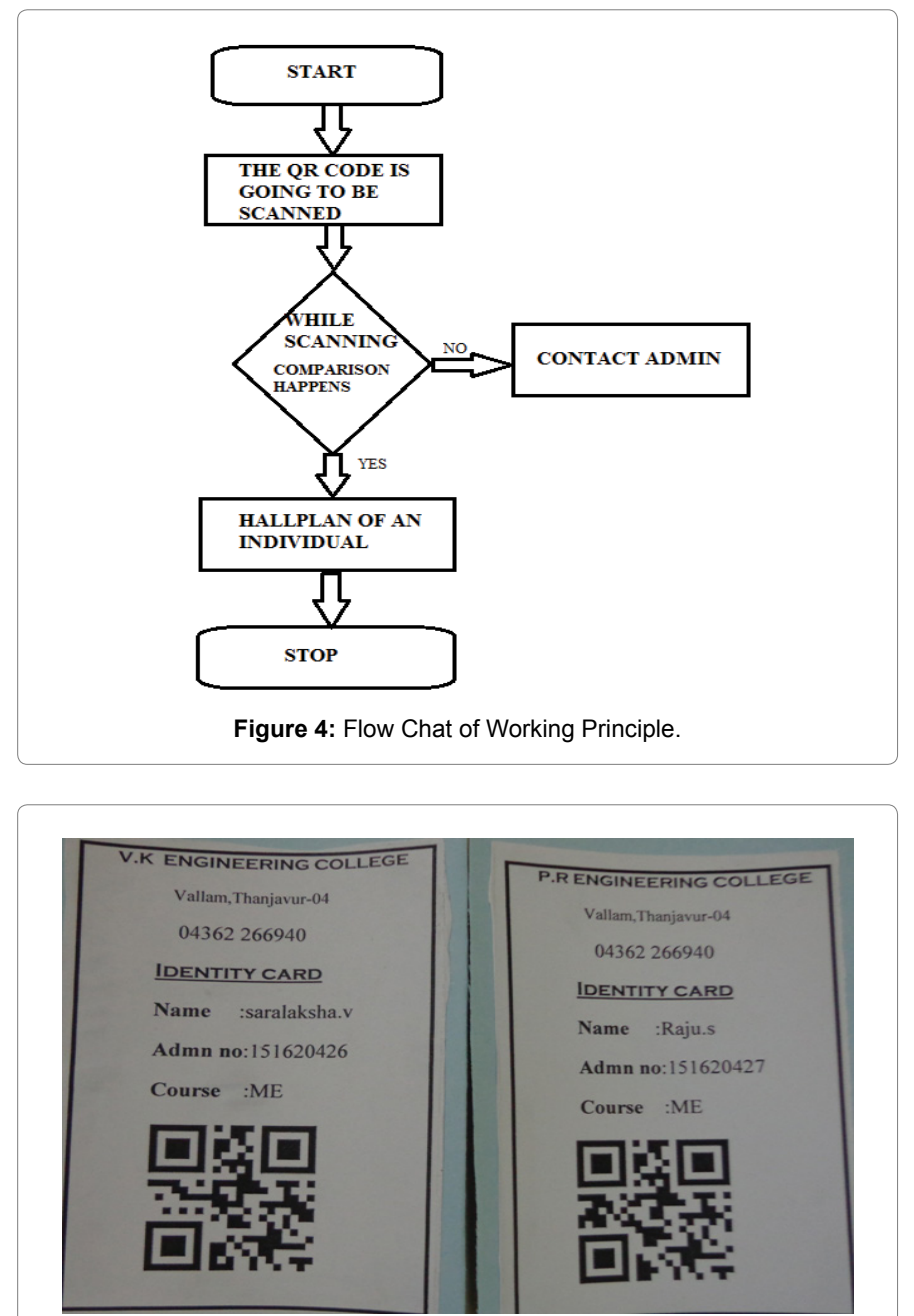

Figure 5: ID-card.

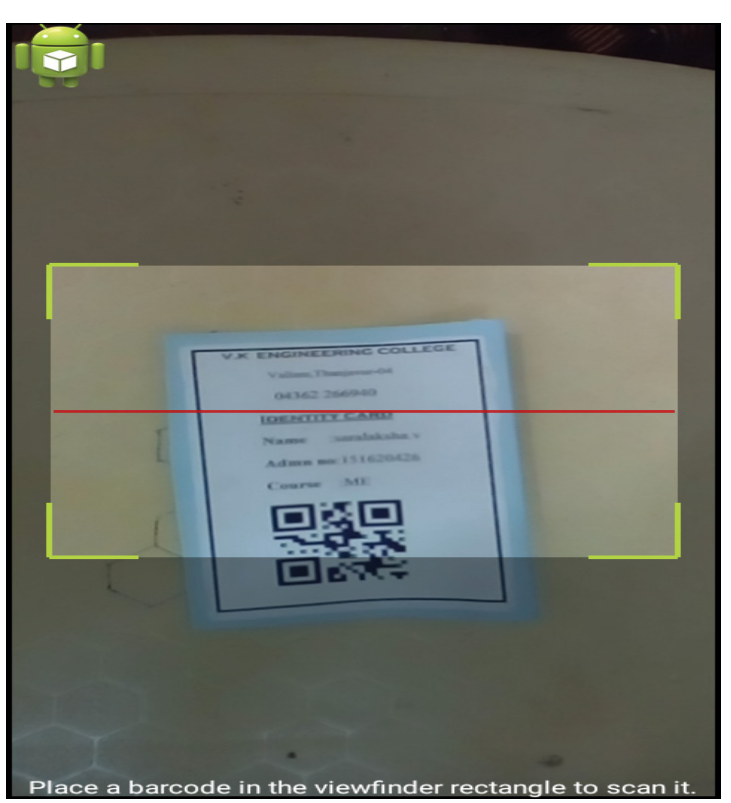

Figure 6: Scanning process. 


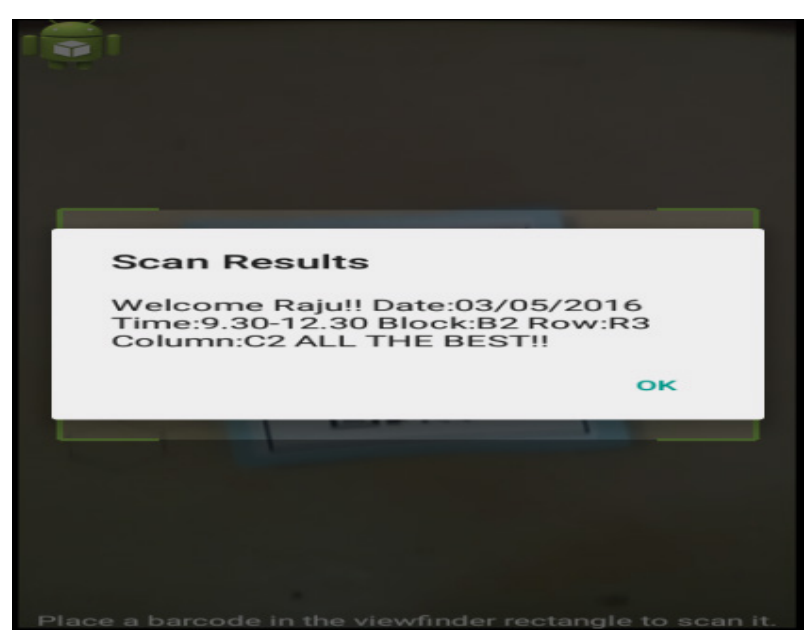

Figure 7: After scanned the correct image.

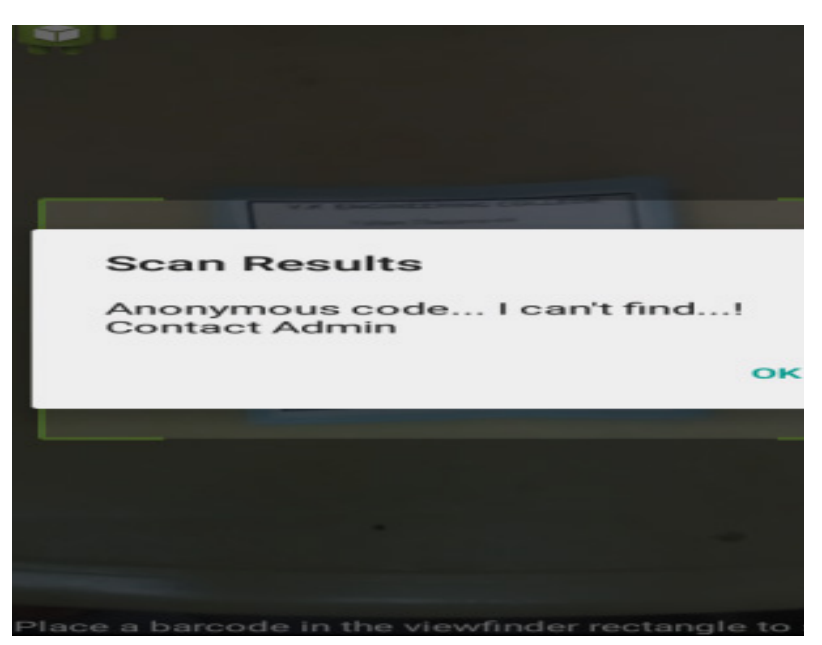

Figure 8: After scanned the incorrect image.

\section{Conclusion}

QR code has many applications as can imagine. In this paper, we have utilized the versatility of QR code in implementing functional and provide the exam hall plan details in an efficient manner. This project helps the user to know the exam details of the respective subjects in a less time and faster. We can add more details about the respective individuals in the future.

\section{References}

1. Liu Y, Liu MY (2005) Research on Data Encoding of Two-Dimensional QR Code Barcode. Trans Beijing Inst Technol 25: 352-355.

2. Zhang H, Han QW, Yu FM Two (2004) Two Dimensional Bar Code in Medicine Trade Logistic Management System. Journal of Chongqing University 27: 122-125.

3. Huang XX (2010) Research on Restaurant Reservation System Based on Two Dimensional Bar Technology China Computer \& Communication Pp: 61-62.

4. Kang CY (2009) Study on System of Electronic Ticket on Two-dimensional Code. J Harbin Univ Commer 25: 178-181.

5. Article Numbering Centre of China, QR code Two-dimensional Bar Code Technology and Application, Beijing, Standards Press of China, Pp: 2-50.

6. Quick response code, G BIT 18284-2000.

7. Trier OD, Jain AK (1995) Goal-Directed Evaluation of Binarization Methods, Pattern Analysis and Machine Intelligence. JEEE Transactions on PAMI 17: 1191-1201.

8. Zhang R, Zhu H, Zhang T, Shen X (2008) A Pre-Processing Method on QR Code Two Dimensional Code Image. Computer Science 35: 146-148. 